\title{
A Spatially Resolved Spectroscopic Study of NGC 7009
}

\author{
S.-G. Luo ${ }^{1} \&$ X.-W. Liu ${ }^{2}$
}

${ }^{1}$ Department of Astronomy, Peking University, Beijing 100871, PRC

${ }^{2}$ Department of Physics and Astronomy, University College London, Gower Street, London WC1E 6BT, UK

\section{Introduction}

Liu et al. (1995) and Luo, Liu \& Barlow (2001) determined the CNONe abundances in NGC 7009 using optical recombination lines (ORLs) and found that they are all approximately a factor of five higher than the corresponding values derived from collisionally excited lines (CELs). We have obtained new deep high resolution long-slit spectra of NGC 7009. The data have been used to map the electron temperature, density and element abundances across the nebula, using both CELs and ORLs. While the electron temperature derived from the [O III] forbidden line ratio, $T_{\mathrm{e}}([\mathrm{O} \mathrm{III}])$, is found to be largely constant, that derived from Balmer jump of $\mathrm{H}$ I recombination spectrum, $T_{\mathrm{e}}(\mathrm{BJ})$, shows large amplitude complicated variations. The ratios of the heavy element abundances derived from ORLs to those deduced from CELs are found to be correlated with the difference between the difference between $T_{\mathrm{e}}([\mathrm{O} \mathrm{III}])$ and $T_{\mathrm{e}}(\mathrm{BJ})$ - a relation previously known to exist from analyses of the integration spectra of individual nebulae (Liu et al. 2001).

\section{Observations and Results}

The data were obtained at the WHT $4.2 \mathrm{~m}$ telescope using the ISIS double spectrograph and at the ESO $1.5 \mathrm{~m}$ telescopes using the B\&C spectrograph. The slit were oriented in $\mathrm{PA}=79 \mathrm{deg}$, parallel to the nebular major-axis and passing through the CS and the two outlying ansae at radii $\sim 25$ arcsec from the CS. The data was processed using the standard method. After integrating the spectra along the slit, we found a logarithmic extinction at $\mathrm{H} \beta c=0.07$, an electron density $N_{\mathrm{e}}=4000 \mathrm{~cm}^{-3}$, an $[\mathrm{O} \mathrm{III}]$ forbidden line temperature $T_{\mathrm{e}}([\mathrm{O} \mathrm{III}])=$ $9980 \mathrm{~K}$, and a Balmer temperature $T_{\mathrm{e}}(\mathrm{BJ})=7100 \mathrm{~K}$.

The variations along the nebular major axis of $N_{\mathrm{e}}, T_{\mathrm{e}}([\mathrm{O} \mathrm{III}]), T_{\mathrm{e}}(\mathrm{BJ})$, the ionic and total abundances of He derived from He I and He II ORLs, as well as $\mathrm{C}^{2+} / \mathrm{H}^{+}, \mathrm{O}^{2+} / \mathrm{H}^{+}$and $\mathrm{N}^{3+} / \mathrm{H}^{+}$derived from ORLs are plotted in Fig. 1. After corrected for small ionization corrections, the total $\mathrm{O} / \mathrm{H}$ abundances derived from CELs and ORLs are also plotted and compared in Fig. 1.

$N_{\mathrm{e}}$ is found to be uniform within the nebular radius $r<14 \operatorname{arcsec}$ and has a value of $\sim 4000 \mathrm{~cm}^{-3}$. Beyond this, it declines rapidly to values below $1000 \mathrm{~cm}^{-3}$. The forbidden line temperature $T_{\mathrm{e}}([\mathrm{O} \mathrm{III}])$ is also found to be largely constant, decreasing only slightly from a value of $\sim 11000 \mathrm{~K}$ at the nebular centre to $\sim 9500 \mathrm{~K}$ in the outer regions. By contrast, $T_{\mathrm{e}}(\mathrm{BJ})$ shows complicated variations. It has a minimum of $\sim 5600 \mathrm{~K}$ at the nebular centre, increases to $\sim 8000 \mathrm{~K}$ between $5<r<15$, and then drops again dramatically $r>15$ arcsec, reaching $\sim 2500 \mathrm{~K}$ in some places. 


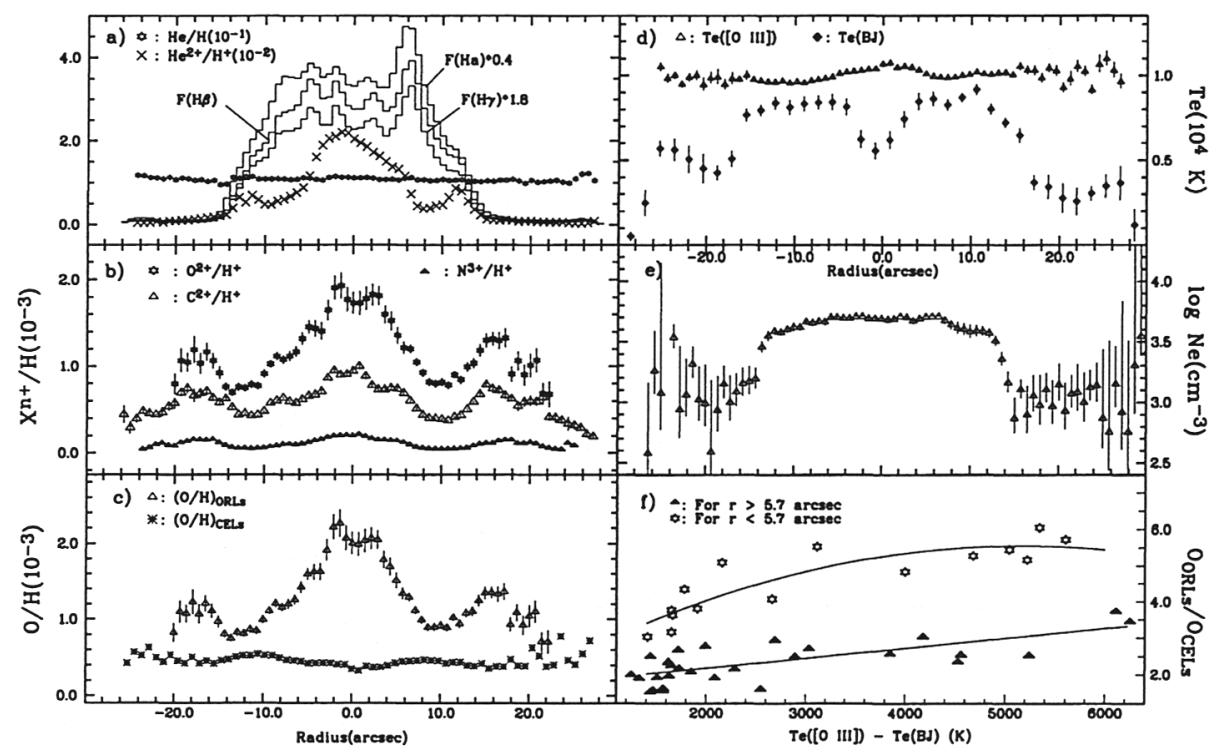

Figure 1. Variations along the nebular major-axis of NGC 7009 of: a) The brightness of $\mathrm{H} \alpha, \mathrm{H} \beta$ and $\mathrm{H} \gamma$ (solid lines), $\mathrm{He} / \mathrm{H}$ (stars) and $\mathrm{He}^{2+} / \mathrm{H}^{+}$(crosses) ratios; b) $\mathrm{O}^{2+} / \mathrm{H}^{+}$(stars), $\mathrm{C}^{2+} / \mathrm{H}^{+}$(triangles) and $\mathrm{N}^{3+} / \mathrm{H}^{+}$(filled triangles) ratios derived from ORLs; c) Elemental $\mathrm{O} / \mathrm{H}$ abundances derived from ORLs (triangles) and from CELs (asterisks); d) $T_{\mathrm{e}}([\mathrm{O} \mathrm{III}])$ (triangles) and $T_{\mathrm{e}}(\mathrm{BJ})$ (filled lozenges); e) $N_{\mathrm{e}}$ derived from the [Ar IV] $\lambda 4740 / \lambda 4711$ ratio. In $\mathrm{f})$, the ratio of $\mathrm{O}^{2+} / \mathrm{H}^{+}$derived from ORLs and CELs is plotted against $T_{\mathrm{e}}([\mathrm{O} \mathrm{III}])-T_{\mathrm{e}}(\mathrm{BJ})$ for the regions of $r>5.7$ (filled triangles) and $r<5.7 \operatorname{arcsec}$ (stars), respectively.

The total $\mathrm{He} / \mathrm{H}$ abundance ratio is found to be constant across the whole nebula. The total $\mathrm{O} / \mathrm{H}$ abundance ratio derived from CELs varies by less than 0.2 dex. On the other hand, for all the ionic species mapped with ORLs, the resultant abundance ratios exhibit similar complicated spatial variations across the nebula, with amplitudes of a factor of 2-4. Liu et al. (2001) found that the ORL to CEL abundance ratios derived for individual nebulae are positively correlated to the differences between $T_{\mathrm{e}}([\mathrm{O} \mathrm{III}])$ and $T_{\mathrm{e}}(\mathrm{BJ})$ observed in those nebulae. In Panel f) of Fig. 1, the ORL to CEL abundance ratios derived at different positions along the nebular major axis are plotted against $T_{\mathrm{e}}([\mathrm{O} \mathrm{III}])-$ $T_{\mathrm{e}}(\mathrm{BJ})$ measured at the same positions. As found previously for individual nebulae, the two quantities are clearly positively correlated. There is also evidence that the ORL to CEL abundance ratio at a given position is correlated with the surface brightness and are the largest for the high surface brightness regions.

\section{References}

Liu, X.-W., Storey, P.J., Barlow, M.J. \& Clegg, R.E.S. 1995, MNRAS, 272, 369

Luo, S.-G., Liu, X.-W. \& Barlow, M. J. 2001, MNRAS, 326, 1049

Liu X.-W., Luo S.-G., Barlow, M. J., et al. 2001, MNRAS, 327, 141 